\title{
Planck Scale Effects on the Majoron
}

\author{
E.Kh. Akhmedov ${ }^{1,2}$, Z.G. Berezhiani ${ }^{3,4}$, R.N. Mohapatra ${ }^{5}$ \\ and G. Senjanović ${ }^{6}$ \\ ${ }^{1}$ Scuola Internazionale Superiore di Studi Avanzati \\ I-34014 Trieste, Italy \\ ${ }^{2}$ Kurchatov Institute of Atomic Energy, Moscow 123182, Russia \\ ${ }^{3}$ Sektion Physik der Universität München \\ D-8000 München, Germany \\ ${ }^{4}$ Institute of Physics, Georgian Academy of Sciences \\ 380077 Tbilisi, Georgia \\ ${ }^{5}$ Department of Physics, University of Maryland \\ College Park, Maryland 20742, U.S.A. \\ ${ }^{6}$ International Centre for Theoretical Physics \\ I-34014 Trieste, Italy
}

\begin{abstract}
The hypothesis that non-perturbative gravitational effects lead to explicit breaking of global symmetries is considered in the context of Majoron models. We find that the nonvanishing Majoron mass generated by these effects can overclose the universe unless the massive Majoron is unstable. The cosmological mass density constraints can then be satisfied only if $V_{B L}<10 \mathrm{TeV}$, where $V_{B L}$ is the scale of $B-L$ symmetry breaking.
\end{abstract}


1. The idea that neutrinos may be massive Majorana particles has been extensively discussed in literature. If this possibility is realized in nature, it would imply that $B-L$ symmetry of the standard model is broken by new interactions beyond the standard model, and will provide a window to this new physics. It was proposed in $1980^{[1]}$ that $B-L$ may be global symmetry of nature spontaneously broken by the vacuum state. In this case, there appears a massless spin zero Nambu-Goldstone boson in the particle spectrum called the Majoron, which significantly alters our thinking about the early universe and the role of neutrinos in its evolution.

The original idea of the Majoron was realized in an extension of the standard model where the $B-L$ symmetry was spontaneously broken by the vacuum expectation value of an electroweak singlet complex scalar boson. The associated Majoron is called the singlet Majoron model ${ }^{[1]}$. Subsequently, this idea was realized in models where the $B-L$ symmetry was spontaneously broken by the vev's of iso-triplet ${ }^{[2]}$ and iso-doublet ${ }^{[3]}$ scalar bosons leading to the so-called triplet and doublet Majoron models, respectively. The measurement of the $Z$-width at LEP has ruled out the triplet and doublet Majoron models. At present, the only viable Majoron model is the singlet model or mixed Majoron ${ }^{[4]}$ models, where tiny admixtures of non-singlet components appear in combination with a dominantly singlet Majoron.

An important question in these models is the scale $V_{B L}$ at which the $B-L$ symmetry breaks. Experimentally, this scale will manifest itself in the Majorana mass of the neutrino. For instance, in the see-saw models for neutrino masses, the neutrino mass scales inversely with $V_{B L}$. In the singlet Majoron model or its generalizations, $V_{B L}$ is an arbitrary parameter. In this letter, we argue that if non-perturbative gravitational effects explicitly break all global symmetries, as has recently been postulated ${ }^{[5,6,7]}$, the scale $V_{B L}$ of the singlet Majoron model (or its extensions) will have an upper limit of about a few $\mathrm{TeV}$, or so.

2. An important ingredient of the singlet Majoron model is a complex Higgs singlet $\sigma$ with $B-L=-2$, which couples to the right-handed neutrino $\nu_{R}$, and acquires a non-zero vev: $\langle\sigma\rangle=V_{B L} / \sqrt{2}$. One can write:

$$
\sigma=\frac{1}{\sqrt{2}}\left(V_{B L}+\rho\right) e^{i \chi / V_{B L}}
$$


In the absence of gravitational effects, $\chi$ is massless and denotes the singlet Majoron field. In the early universe, for $T>>V_{B L},<\sigma>$ vanishes and the Majoron field acquires a mass. In the mixed Majoron models, the true Majoron field has only $\chi$ as the dominant piece, and the discussion given below applies.

Once the gravitational effects are turned on, $U(1)_{B-L}$ breaking terms will be induced. We assume that these terms respect the local symmetries of the theory and vanish as $M_{P \ell} \longrightarrow \infty$. One can then write these induced terms that involve only the singlet field $\sigma$ and the standard model Higgs doublet $\varphi$, in which case the lowest dimension terms are $d=5$ :

$$
V_{P \ell}=V_{1}(\sigma)+V_{2}(\varphi, \sigma)
$$

where

$$
\begin{gathered}
V_{1}(\sigma)=\lambda_{1} \frac{\sigma^{5}}{M_{P \ell}}+\lambda_{2} \frac{\sigma^{*} \sigma^{4}}{M_{P \ell}}+\lambda_{3} \frac{\sigma^{*^{2}} \sigma^{3}}{M_{P \ell}}+h . c . \\
V_{2}(\varphi, \sigma)=\beta_{1} \frac{\left(\varphi^{\dagger} \varphi\right)^{2} \sigma}{M_{P \ell}}+\beta_{2} \frac{\left(\varphi^{\dagger} \varphi\right) \sigma^{2} \sigma^{*}}{M_{P \ell}}+\beta_{3} \frac{\left(\varphi^{\dagger} \varphi\right) \sigma^{3}}{M_{P \ell}}+\text { h.c. }
\end{gathered}
$$

We will further assume that $\lambda$ and $\beta$ are not small. $V_{P \ell}$ will now induce non-vanishing mass for the Majoron field $\chi$. A massive Majoron will affect the evolution of the universe, unless its mass and lifetime satisfy certain constraints. This, in turn, leads to restrictions on the value of the $B-$ $L$ breaking scale $V_{B L}$. In order to discuss this question, we consider two complementary ranges of the parameter $V_{B L}$ :

(A) $V_{B L}<V$ and (B) $V_{B L}>V$, where $V=\left(\sqrt{2} G_{F}\right)^{-\frac{1}{2}} \simeq 246 \mathrm{GeV}$. In case (A), the Majoron mass is dominated by the $\beta_{1}$-term in eq. (3), whereas in case (B), the dominant terms are from $\lambda_{1}, \lambda_{2}$, and $\lambda_{3}$ couplings. In case (A), the Majoron mass is estimated to be (estimation valid for $m_{\chi} \leq V_{B L}$

$$
m_{\chi} \simeq \beta_{1}^{\frac{1}{2}}\left(\frac{V}{V_{B L}}\right)^{\frac{1}{2}} \mathrm{keV}
$$

On the other hand, in case $(\mathrm{B})$, we get

$$
m_{\chi} \simeq\left(\frac{25}{2} \lambda_{1}+\frac{9}{2} \lambda_{2}+\frac{1}{2} \lambda_{3}\right)^{\frac{1}{2}}\left(\frac{V_{B L}}{V}\right)^{\frac{3}{2}} \mathrm{keV}
$$


Obviously, the mass acquired by the Majoron is minimal when $V_{B L}=V$. We see that if $\lambda$ 's and $\beta$ 's are of order 0.1 to 1 , the Majoron mass is larger than a $\mathrm{keV}$. Therefore, unless its lifetime is appropriately constrained, the Majoron population of the universe will overclose it. As a rough estimate of the situation, we see from the desired constraint

$$
n_{\chi} m_{\chi}<\rho_{\text {crit }}
$$

that, if $n_{\chi} \simeq n_{\gamma}$, then for $V=V_{B L}$, we must have $\lambda, \beta<10^{-2}$. As $V_{B L}$ becomes significantly different from $V$, the situation is worse and the Majoron must be unstable. Of course, if it happens that $\chi$ decouples sufficiently early, leading to $n_{\chi}<<n_{\gamma}$, constraints on $\chi$ may be weaker. To study the implications of a massive Majoron for cosmology, we therefore need to know the following:

i) When does the Majoron go out of equilibrium?

ii) How does it decay?

Both these questions are tied to the nature of interactions that involve the $\sigma$-field. Typically, the following interaction terms dictate the answers to the above questions:

$$
L_{Y}=h_{a b} \bar{\psi}_{a L} \varphi \nu_{i R}+f_{a b} \nu_{a R}^{T} C^{-1} \nu_{b R} \sigma+\text { h.c. }
$$

Here $\psi_{a L}$ denotes the leptonic doublet, $\nu_{a R}$ is the right-handed neutrino field and $a, b, \ldots$ stands for the generation index. Eq. (7) leads to the see-saw formula for neutrino masses when we substitute $\left\langle\varphi^{o}\right\rangle=\frac{V}{\sqrt{2}}$ and $<\sigma>=\frac{V_{B L}}{\sqrt{2}}$, which yields:

$$
h \approx \frac{m_{\nu}^{D}}{V} \sqrt{2} \approx \frac{\sqrt{2 m_{\nu} m_{\nu_{R}}}}{V}
$$

(Here and below, we consider only the heaviest of the neutrinos, denoted by $\nu$, since only its effects are the most significant). Since in our framework the Majorons are massive, the left-handed neutrinos are stable and cosmological mass density constraint requires that $m_{\nu}<25 \mathrm{eV}$. This implies that

$$
h \leq 10^{-6}\left(\frac{m_{\nu_{R}}}{\mathrm{GeV}}\right)^{\frac{1}{2}}
$$


It is then easy to see that $\nu_{R}$ is in equilibrium with the left-handed electrons and neutrinos via the interaction $\psi_{L}+\phi \longrightarrow \nu_{R}+W_{L}$ (which has an interaction rate $\Gamma$ given by $\Gamma \approx \frac{g^{2} h^{2}}{16 \pi} T$ ), unless $m_{\nu} \leq 10^{-4} \mathrm{eV}$.

This is in equilibrium for $10^{5} m_{\nu_{R}} \geq T>m_{\nu_{R}}$. Since the $\sigma$-field is in equilibrium with the right-handed neutrinos via the Yukawa coupling $f$ (if the latter is not unnaturally small), they are in equilibrium with the rest of the universe. As the universe cools below $T=m_{\nu_{R}}$, the right-handed neutrinos disappear. The dominant interactions of $\sigma$ is via its induced coupling to $\nu_{L}$ 's. This coupling has a magnitude

$$
f_{\nu_{L} \nu_{L} \sigma} \simeq\left(\frac{m_{\nu}}{V_{B L}}\right)
$$

For $V_{B L}$ in the $1 \mathrm{GeV}$ to $100 \mathrm{GeV}$ range, $f_{\nu_{L} \nu_{L} \sigma} \simeq 2 \times 10^{-8}-2 \times 10^{-10}$. Therefore, for $m_{\nu_{R}}>10 \mathrm{GeV}$ or so, $\sigma$ 's go out of equilibrium at $T=m_{\nu_{R}}$. Their present density is then given by

$$
r_{\chi}=\frac{n_{\chi}\left(T_{o}\right)}{n_{\gamma}\left(T_{o}\right)}=\frac{g_{*}\left(T_{o}\right)}{g_{*}\left(T_{D}\right)} \approx \frac{1}{10}-\frac{1}{20}
$$

In eq. $(11), g_{*}(T)$ denotes the effective number of light particle species at temperature $T ; T_{D}$ and $T_{o}$ denote the decoupling temperature for the righthanded neutrinos and the present temperature of the universe respectively.

Eq. (6) then implies that $m_{\chi}<2 \mathrm{keV}$. Using eq. (4) and (5), one finds for all $\lambda$ 's and $\beta$ 's of the order one, that neither case (A) nor case (B) are acceptable unless the Majoron decays. On the other hand, if $\lambda_{i}<10^{-1}$, then $V_{B L} \simeq V$ is acceptable for the $B-L$ symmetry breaking scale.

3. Now let us look at the constraints on $V_{B L}$ arising when the Majoron is unstable. According to eq. (10), the dominant decay mode, $\chi \rightarrow \nu \nu$, is provided by the heaviest of the ordinary neutrinos. Then the lifetime is given by

$$
\tau_{\chi}=8 \pi\left(\frac{V_{B L}}{m_{\nu}}\right)^{2} m_{\chi}^{-1}
$$

The constraint to be satisfied in this case is ${ }^{[8]}$ 


$$
r_{\chi} m_{\chi}\left(\frac{\tau_{\chi}}{\tau_{U}}\right)^{\frac{1}{2}}<25\left(\Omega_{o} h^{2}\right) \mathrm{eV}
$$

where $\tau_{U}$ is the age of the universe. This leads to

$$
r_{\chi}\left(\frac{m_{\chi}}{1 \mathrm{keV}}\right)\left(\frac{\tau_{\chi}}{1 \mathrm{sec}}\right)^{1 / 2} \leq 10^{7} \Omega_{o} h^{\frac{3}{2}}
$$

A more stringent constraint comes from the requirements of galaxy formation in the universe. The recent COBE measurements of cosmic background anisotropy ${ }^{[9]}$ implies a very small magnitude for initial density fluctuations: $\delta \rho / \rho \leq 10^{-4}$. This, in turn, requires a sufficiently long matter dominated epoch for the linear growth of $\delta \rho / \rho$ to form the observed large scale structure of the universe. Therefore, the relativistic decay products of $\chi$ must be redshifted enough so as to have a matter dominated universe by the era, $t_{e q}$, where one has the equality of matter and radiation density ${ }^{[10]}$ $\left(t_{e q}=0.8 \times 10^{3}\left(\Omega h^{2}\right)^{-2} \mathrm{yr}\right)$. This leads to

$$
r_{\chi} n_{\gamma}\left(t_{e q}\right) m_{\chi} \cdot\left(\frac{\tau_{\chi}}{t_{e q}}\right)^{\frac{1}{2}}<\rho_{M}\left(t_{e q}\right)
$$

where $n_{\gamma}\left(t_{e q}\right)=\left(1+z_{e q}\right)^{3} \times 422 \mathrm{~cm}^{-3}$ is the photon number density at that epoch and $\rho_{M}\left(t_{e q}\right)=\left(1+z_{e q}\right)^{3} \times 10.5\left(\Omega_{o} h^{2}\right) \mathrm{keV} \cdot \mathrm{cm}^{-3}$ is the energy density of non-relativistic (presumably cold dark) matter. This leads to the condition:

$$
r_{\chi}\left(\frac{m_{\chi}}{1 \mathrm{keV}}\right)\left(\frac{\tau_{\chi}}{1 \mathrm{sec}}\right)^{\frac{1}{2}}<4 \times 10^{3}
$$

(Notice that this condition does not depend on the value of $\Omega_{o} h^{2}$ ). Assuming $r_{\chi}=0.1$, this can be rewritten as

$$
m_{\chi}\left(\frac{V_{B L}}{V}\right)^{2} \leq 10^{6}\left(\frac{m_{\nu}}{25 \mathrm{eV}}\right)^{2} \mathrm{keV}
$$

Using eq. (5) and assuming the numerical factors in it to be of order one, we get:

$$
V_{B L}<\left(\frac{m_{\nu}}{25 \mathrm{eV}}\right)^{4 / 7} \times 10 \mathrm{TeV}
$$


If we use eq. (4) instead of eq. (5), the result is an upper limit on $V_{B L}$ bigger than the electro-weak scale (where eq. (4) does not hold). We thus conclude that the limit on the scale of $B-L$ breaking due to Planck scale effects inducing $B-L$ breaking terms of dimension 5 , is given by eq. (18) and is in the $\mathrm{TeV}$ range. The limit actually does not depend on the particular model of the singlet Majoron and applies to the original see-saw model ${ }^{[1]}$ as well as the modified versions ${ }^{[4]}$.

An important implication of our result is that the neutrino masses must have a lower bound if they arise via the see-saw formula. The precise value of the lower bound, of course, depends on the magnitude of the Dirac mass of the neutrino. In particular, since the $B-L$ scale is so low, one of the neutrinos could have a mass in the $\mathrm{eV}$ range which in turn implies that a few per cent of the dark matter of the universe being hot. This may indeed be indicated by the recent COBE data.

4. Our considerations could easily be extended to a wider class of theories where the leading order $B-L$ violating term may be bigger than 5 . For instance, if the leading order term is of the form $\frac{\sigma^{4+n}}{M_{P \ell}^{n}}$, then

$$
V_{B L}<(10)^{\frac{10}{n+6}}\left(\frac{M_{P \ell}}{\mathrm{GeV}}\right)^{\frac{n}{n+6}}\left(\frac{m_{\nu}}{25 \mathrm{eV}}\right)^{\frac{4}{n+6}} \mathrm{GeV}
$$

Thus, for higher $n$, bounds on $V_{B L}$ are less stringent. It is possible to construct gauge models where the Planck scale effects can be postponed to higher dimensional terms. We do not discuss specific models in this paper.

In conclusion, we point out that Planck scale effects can impose interesting upper limits on the scale of $B-L$ breaking in Majoron models. These considerations can be easily extended to familon and other models with spontaneously broken global symmetries, where the pseudo-Goldstone bosons couple to neutrinos.

\section{Acknowledgements}

We would like to thank G. Fiorentini, E. Bellotti and the organizers of the Gran Sasso Neutrino Workshop where this work was initiated and A. Dolgov, 
A. Smirnov and M. Vysotsky for useful discussions. After this work was completed, we were informed that similar ideas are also under consideration by K.S. Babu, I.Z. Rothstein, and D. Seckel (to appear as Bartol preprint). The work of R.N.M. is supported by a grant from the National Science Foundation and that of Z.B. is supported by the Alexander von Humboldt Foundation.

\section{References}

[1] Y. Chikashige, R.N. Mohapatra and R.D. Peccei, Phys. Lett. 98B, 265 (1981).

[2] G. Gelmini and M. Roncadelli, Phys. Lett. 99B, 411 (1981); H. Georgi, S.L. Glashow and S. Nussinov, Nucl.Phys. B193, 297 (1981).

[3] C. Aulakh and R.N. Mohapatra, Phys. Lett. 119B, 136 (1983); S. Bertolini and A. Santamaria, Nucl.Phys. B310, 714 (1988).

[4] K.S. Babu and R.N. Mohapatra, Phys. Rev.Lett. 67, 1498 (1991); K. Choi and A. Santamaria, San Diego Preprint (1991); A. Joshipura, PRL Preprint (1991); Z. Berezhiani, A.Yu. Smirnov and J.W.F. Valle, Preprint FTUV/92-20 (1992).

[5] S. Giddings and A. Strominger, Nucl. Phys. B307, 854 (1988); S. Coleman, Nucl. Phys. B310, 643 (1988); S.J. Rey, Phys. Rev. D39, 3185 (1989); B. Carter, in General Relativity, "An Einstein Centenary Survey", ed. by S. Hawking and W. Israel, Cambridge University Press, (1979).

[6] R. Barbieri, J. Ellis and M.K. Gaillard, Phys. Lett. 90B, 249 (1980); E. Akhmedov, Z. Berezhiani and G. Senjanović, preprint IC/92/79, (1992).

[7] R. Holman, S. Hsu, T. Kephart, E. Kolb, R. Watkins and L. Widrow, Phys. Lett. 282B 132 (1992); M. Kamionkowski and J. March-Russel, Phys. Lett. 282B, 137 (1992); S. Barr and D. Seckel, Phys. Rev. D46, 539 (1992); D. Grasso, M. Lusignoli and M. Roncadelli, Phys. Lett. 288B, 140 (1992); S. Ghigna, M. Lusignoli and M. Roncadelli, Phys. Lett. 283B, 278 (1992). 
[8] D. Dicus, E. Kolb, V. Teplitz and R. Wagoner, Phys. Rev. D18, 1829 (1978).

[9] G.F.Smoot et. al., Ap. J. Lett. (in press) (1992).

[10] G. Steigman and M. Turner, Nucl. Phys. B253, 375 (1985). 